\title{
The Nature of Light: What are "Photons"?*
}

\author{
Carver Mead \\ California Institute of Technology, Pasadena, CA 91125, USA
}

Keywords: photon, quantum, transaction, coherent, collective, electrodynamics, electromagnetism

\section{PHYSICAL LAW}

We are told that our present understanding of physical law was ushered in by the Quantum Revolution, which began around 1900 and was brought to fruition around 1930 with the formulation of modern Quantum Mechanics. The "photon" was supposed to be the centerpiece of this revolution, conveying much of its conceptual flavor. What happened during that period was a rather violent redirection of the prevailing world view in and around physics - a process that has still not settled. In this paper I critically review the evolution of the concepts involved, from the time of Maxwell up to the present day.

At any given time, discussions in and around any given topic take place using a language that presupposes a world view or zeitgeist. The world view itself limits what ideas are expressible. We are all prisoners of the language we have created to develop our understanding to its present state. Thus the very concepts and ways of thinking that have led to progress in the past are often the source of blind spots that prevent progress into the future. The most insidious property of the world view at any point in time is that it involves assumptions that are not stated. In what follows we will have a number of occasions to point out the assumptions in the current world view, and to develop a new world view based on a quite different set of assumptions.

\subsection{The Present World View}

Today's scientific thinking is dominated by a number of underlying assumptions, spoken or unspoken. A few of them are:

1. Time has a preferred direction.

2. Isolated experiments are the key to fundamental physical quantities.

3. Space-Time has degrees of freedom of its own.

4. Matter and fields are particulate in nature.

5. The most fundamental constructs in physics are particles manifest in high-energy experiments. These fundamental elements of matter are point particles.

6. Mathematical elegance is the most important attribute of a theory.

7. The mathematics of a theory constitutes the entire theory.

8. It is not necessary for a theory to enable conceptual reasoning.

9. It is acceptable for a theory to be logically inconsistent.

10. Theory often predicts new physical phenomena.

11. The successes in technological enterprise are attributable to current theoretical understanding.

Once these prejudices are articulated, it becomes clear that not every person subscribes to all of them. Some seem self-evident, some seem irrelevant, and some seem nonsense to any given person. Yet no two people, even if they are working in the same discipline, agree on which is which. And all of these ideas are, in one way or another, effectively shaping current scientific discussion.

${ }^{*}$ This paper is part of a revision effort for Collective Electrodynamics,${ }^{1}$ hereinafter referred to simply as CE. 


\subsection{Alternate World View}

As strange as it may seem, the physical world, as we observe it, can be much more simply and elegantly understood using a quite different set of assumptions:

1. The most fundamental constructs in physics are the electromagnetic and gravitational interactions of matter.

2. Time enters these fundamental physical interactions in a totally symmetric way. The apparent preferred direction of time is thermodynamic in origin.

3. Every element of matter is coupled to all other matter on its light cone by time-symmetric interactions.

4. Matter interacts directly with other matter.

The time-symmetric nature of these interactions make them effectively instantaneous.

5. The properties of matter, such as its rest energy, its inertia, its radiation reaction, its spontaneous emission, and other properties as well, are manifestations of these interactions. There is no such thing as an isolated experiment.

6. Fields have no degrees of freedom of their own.

They are simply bookkeeping mechanisms for the direct interaction of matter with other matter.

7. Space-Time has no degrees of freedom of its own.

It is simply a coordinate system within which we can express physical law.

8. Matter is, at the fundamental level, wavelike in nature. There is no such thing as a point particle. Quantization of matter is due to the formation of bound states of matter wave functions.

9. Quantization of quantum transition energies is due to the discreteness of these bound matter states. The fields themselves are simply a representation of the matter interactions.

They confer no additional constraints on those interactions.

10. A "photon" can be best viewed as a transaction between two atoms on the same light cone. Such a transaction requires an exquisite degree of phase matching between the quantum states of the two coupled atoms, and is therefore a rare event. Photon statistics result from the chance correlations of atomic wave function phases.

11. The "photon" transaction can be viewed as a brief entanglement of the quantum states of the two participating atoms.

12. The transaction is itself a completely continuous process. When a transaction is initiated, it grows exponentially with time. This highly nonlinear evolution gives the appearance of an abrupt "quantum jump" as the atomic wave functions "collapse".

13. The observed particulate "photon" behavior is not due to the nature of fields, but is a manifestation of entropy, related to the thermodynamic arrow of time.

14. It is centrally important for a theory to be logically consistent.

15. Mathematical elegance is important in a theory when it aids conceptual reasoning. It is destructive when it obscures otherwise accessible conceptual understanding.

16. It is rare for theory to predict new physical phenomena. In most cases each new phenomenon is discovered/invented experimentally, and the current theory is quickly "adjusted" so as to "predict" the observed result.

17. The successes in technological enterprise are attributable to conceptual reasoning. "Fundamental" physical theory, as taught in universities and and propagated in the literature, has a long history of hindering conceptual reasoning, and therefore technological progress. 


\subsection{Macroscopic Quantum Systems}

It has been known since the historic experiments of Davisson and Germer that elements of matter have wavelike properties. As experiments progressed, it became more and more clear that all "particles" had wave properties. The Copenhagen school of thought relegated these properties to the statistical outcome of experiments. A direct result of the statistical view of quantum mechanics was the unspoken assumption that all aggregations of matter would be statistical averages, and would therefore not exhibit wave properties. The first clue that this statistical view of aggregate matter was not correct was the 1933 observation of persistent current in superconducting rings. If the macroscopic electrical current was some kind of statistical average, there was no conceivable way it could persist indefinitely. Fritz London was quick to grasp the quantum implications of persistent current, and predicted

1. Electrons in a superconductor formed a coherent collective state with a well-defined wave function.

2. Persistent currents were stable because the wave function phase must be continuous around the ring.

3. The magnetic flux in the ring should therefore be quantized.

It was not until 1961 that quantization of flux in superconducting rings was observed experimentally.

It showed, for once and for all time, that the degrees of freedom of the field and the degrees of freedom of the charges do not represent different degrees of freedom, but are simply two different representations of the same degrees of freedom.

As Einstein put it ${ }^{\dagger}$ :

I feel it is a delusion to think of the electrons and the fields as two physically different, independent entities.

Since neither can exist without the other, there is only one reality to be described

which happens to have two different aspects;

and the theory ought to recognize this from the start

instead of doing things twice.

Wheeler and Feynman were even more blunt:

There is no such concept as "the field"

an independent entity with degrees of freedom of its own.

Following the Einstein-Wheeler-Feynman dictum requires us to re-conceptualize quantum physics and electromagnetism as a single, unified discipline. Doing so constrains our conceptual framework extremely tightly: It eliminates many of the intellectual loopholes and "white lies" that are an integral part of traditional treatments. The result is the treatment given in CE, leading to the Alternate World View described above.

\section{A BRIEF HISTORY}

From a historical perspective, it is not difficult to see why the theory grew up the way it did

Maxwell published his monumental Treatise on Electricity and Magnetism in 1873. The theory was recast into the modern vector-calculus form of "Maxwell's Equations" by Oliver Heaviside in 1884. Maxwell himself had observed that his equations had propagating solutions, and guessed that they represented light waves. Radio waves propagating in free space were first observed experimentally, and were shown to travel at a velocity close to that of light, by Heinrich Hertz in 1888. By this time the entire range electromagnetic phenomena appeared to have a solid theoretical base, well supported by experiments at the macroscopic level.

A decade later conceptual problems began to emerge, first with the spectrum of hot isothermal cavities. The thermodynamic principle of equipartition of energy should have allocated equal energy to every electromagnetic

\footnotetext{
${ }^{\dagger}$ Quotations not given explicit references here are referenced in detail in CE.
} 
mode of the cavity, which would lead to infinite energy at shorter and shorter wavelength: "The Ultraviolet Catastrophe." What was observed was an exponential cutoff at short wavelength. Something Was Going On! To understand the phenomenon, Max Planck had to resort to what he considered "an act of despair." He later recalled "I was ready to sacrifice any of my previous convictions about physics", so he made the assumption that the energy of each mode was not a continuous, infinitely divisible quantity, but was a discrete quantity composed of an integral number of finite "energy elements". That conceptual leap was destined to revolutionize physics. Applying it to the observed spectrum, he showed that the "energy element" must be a constant $h$ times the frequency of the mode: the first version of what is now termed Planck's relation. It is important to notice that Planck's relation says nothing about the origin of the quantization: It could be a property of the electromagnetic modes of the cavity. or it could result from the atomic interactions at the walls of the cavity.

That distinction will become crucial later in the story.

In 1905 Einstein introduced the possibility that the radiation field itself could be quantized:

According to the assumption to be contemplated here, when a light ray is spreading out from a point, the energy is not distributed continuously over ever-increasing spaces, but consists of a finite number of energy quanta that are localized in points in space, move without dividing, and can be absorbed or generated only as a whole.

The unspoken assumption here is that the radiation field has degrees of freedom separate from those of the emitting and absorbing atoms, in stark contrast to the earlier Einstein statement.

Nonetheless, the Genie was out of the pot: The "photon", as it came to be known, became a little classical bullet carrying a discrete quantity of energy. While this visualization appeals to classical mechanical thinking, it has led to more than a century of vast conceptual chaos.

By 1909 Einstein had second thoughts, and put forward a deeply penetrating question:

Would it not be possible to replace the hypothesis of light quanta by another assumption that would also fit the known phenomena? If it is necessary to modify the elements of the theory, would it not be possible to retain at least the equations for the propagation of radiation and conceive only the elementary processes of emission and absorption differently than they have been until now?

In 1913 Niels Bohr introduced his first approach to what is now called "the old quantum theory". The theory was not well defined, but was able to provide a classical conceptual basis for many of the observed properties of the emission spectral lines of excited gasses. Many physicists worked on improving and extending the theory, with some notable successes. A central conceptual aspect of the theory that has survived is that "quantum jumps" between energy states of atoms, give rise to emitted "photons". Efforts on the old quantum theory persisted well into the 1920's. In the mid-to-late 1920's, the work of DeBroglie, Schrdingier, Heisenberg, Born, Pauli, and Dirac led from the old quantum theory to the quantum theory that is taught in schools today. Experiments during that formative period were still based on spectra from excited gasses, and hence were, by their very nature, only statistical in nature. The limitations of those experiments were built into the conceptual foundations of the new quantum mechanics, and remain there to this day. In fact, the limitation to statistical predictions was turned into a religion by Bohr and Heisenberg.

They championed the notion that "there was no deeper level to be understood".

But, fortunately, there were those who took Einstein's question seriously.

Gilbert N. Lewis, the physicist and chemist who first conceived what we now call the covalent bond, also coined the term "photon." In 1926 he had this to say about Einstein's question:2

It is generally assumed that a radiating body emits light in every direction, quite regardless of whether there are near or distant objects which may ultimately absorb that light; in other words that it radiates "into space"...

I am going to make the contrary assumption that an atom never emits light except to another atom... 
I propose to eliminate the idea of mere emission of light and substitute the idea of transmission, or a process of exchange of energy between two definite atoms...

Both atoms must play coordinate and symmetrical parts in the process of exchange...

In a pure geometry it would surprise us to find that a true theorem becomes false when the page upon which the figure is drawn is turned upside down. A dissymmetry alien to the pure geometry of relativity has been introduced by our notion of causality.

Thus the man who named the "photon" gave us the first hint of what a sensible one might be like, based on the Transactional View of Quantum Transitions. The transactional approach was the inspiration for Dick Feynman's Thesis, and two papers published by Wheeler and Feynman in the 1940's. In the 1980's it was developed into the Transactional Interpretation of Quantum Mechanics by John Cramer. ${ }^{5}$

This interpretation resolves many thorny conceptual inconsistencies that have plagued the field since the time of Bohr and Heisenberg. Many observed quantum phenomena, such as those associated with Bell's Inequality and related forms of quantum non-locality, are totally incomprehensible from the point of view of "photons", but are easily and naturally understood when viewed as transactions. Cramer's web site ${ }^{6}$ has accessible versions of his most important papers, in which many of these "paradoxes" are discussed.

It would seem that, by now, the transactional view would have become the standard approach to understanding quantum systems, and would be presented and compared with traditional "Copenhagen" approaches in every course on the subject. Sadly, that has not happened. The biggest stumbling block to adoption of this world view seems to be that it requires us to adopt time-symmetric exchange as the fundamental interaction of quantum systems. For some reason, that conceptual step seems insurmountable to those with a committed classical-mechanics mindset.

\subsection{Symmetrical View of Time}

The notion that time has a preferred direction has deep roots: Early attempts at physical law required a constant force to keep an object in motion. This view held sway until Galileo showed that, once objects were put in motion, they would stay in motion unless slowed down by an outside force. Using the assumption of one-way propagation of electromagnetic waves, Abraham and Lorentz showed that an accelerated charge radiated away energy and therefore experienced a damping force. Ritz explicitly attributed the direction of time's arrow to the one-way direction of electromagnetic radiation. It seemed natural to him to put the energy being radiated into the "degrees of freedom of the Ether," and thereby not to worry about it any more.

In all of the Transactional views of quantum interactions, it is essential to use both advanced and retarded solutions to the wave-propagation equations; in other words, to use solutions propagating both forward and backward in time, on an equal footing. The first direct discussion of the principles involved dates back to a joint paper published by Ritz and Einstein in 1909, in which Ritz took the position that only forward-propagating solutions were possible, while Einstein claimed that both forward and backward propagating solutions were equally valid, and the apparent unidirectional nature of time in common experience was due to thermodynamics.

In commenting on his collaboration with Feynman, Wheeler had this to say:

The startling conclusion that Dick Feynman and I reached, which I still believe to be correct, is that if there were only a few chunks of matter in the universe...the future would, indeed, affect the past. What prevents this violation of common sense and experience is is the presence in the universe of a nearly infinite number of other objects containing electrical charge, all of which can participate in a grand symphony of absorption and emission of signals going both forward and backward in time.

Einstein was quite favorably impressed by the Wheeler-Feynman work, and had this comment:

I have always believed that electrodynamics is completely symmetric between events running forward and events running backward in time. There is nothing fundamental in the laws that makes things run in only one direction. The one-way flow of events that is observed is of statistical origin. It comes about because of the large number of particles in the universe that can interact with each other. 
A physical picture of how Wheeler's "grand symphony" actually operates on Einsteins "large number of particles" was put on a firm basis by the Fluctuation-Dissipation Theorem, first established by Nyquist ${ }^{3}$ and later expanded and generalized by Callen \& Welton. ${ }^{4}$ This theorem is one of the most profound findings in all of physics. In section 6 of their paper, Callen \& Welton treat the Abraham-Lorentz damping in detail, and show that the dissipation of energy attributed to this "local law" actually arises from the interaction between the local system and a continuum of closely-spaced quantum levels in the universe as a whole.

According to the direct-action viewpoint of $\mathbf{C E}$ p.94, we can interpret this theorem in the following way: Any dynamical system is coupled to distant matter in the universe, both electromagnetically and gravitationally; there is no such thing as an "isolated experiment." The smooth, continuous nature of the Cosmic Microwave Background (CMB) tells us that we can view this distant matter as a continuum of quantum levels. All "radiation resistance" damping processes are a result of interaction with this matter - no other mechanisms are needed. Local dynamical energy coupled to this matter excites the continuum in a huge number of random ways-random because of the random phases of the wave functions of the distant matter. The number of degrees of freedom in the continuum is vast, and there are exponentially many ways to distribute the energy. Thus entropy is created by any such interaction, and therefore energy is lost from the local dynamical system. This energy loss is strictly in the direction of entropy flow, and has no relation whatsoever to the direction of signal propagation:

If our local system is started at rest, it will approach a level of oscillation by excitation from the CMB with exactly the same time constant with which its energy decays from a more excited state.

So, Finally there is no more need for any modern equivalent of "force needed to keep an object in motion". At the fundamental level, every interaction is bidirectional.

\subsection{The "Photon"}

So what happened to "the photon": Einstein's little bullet carrying a quantum of energy?

Many modern accounts place this concept at the center of "quantum mechanics" and credit it with our entire modern optical "photonic" technology. Surely we need it for something!

In his epic book on Lasers, Tony Siegman has this to say:

We have hardly mentioned photons yet in this book. Many descriptions of laser action use a photon picture...in which billiard-ball-like photons travel through the laser medium. Each photon, if it strikes a lower-level atom, is absorbed and causes the atom to make a "jump" upward. On the other hand, a photon, when it strikes an upper-level atom, causes that atom to drop down to the lower level, releasing another photon in the process. Laser amplification then appears as a kind of photon avalanche process.

Although this picture is not exactly incorrect, we will avoid using it to describe laser amplification and oscillation, in order to focus from the beginning on the coherent nature of the stimulated transition process. The problem with the simple photon description...is that it leaves out and even hides the important wave aspects of the laser interaction process...the whole stimulated transition process should be treated, not as a "photon process" but as a coherent wave process.

So there we have it in a nutshell - the concept of a little bullet of quantized energy actively obscures what would otherwise be simple to understand at the conceptual level.

It is one of the last remnants of classical mechanics doing violence to an otherwise coherent discipline.

There is, however, another quite different way of visualizing the photon that has a very real place in understanding coherent systems: In all the recent Bose-Einstein work, intense laser beams are used to cool collections of atoms to nano-Kelvin temperatures. If those beams were made up of Copenhagen "photons", the "photon shot noise" would increase as the square root of the intensity. What actually happens is that the cooling improves as we increase the laser-beam intensity up to a point, after which no further improvement is observed. The point at which this limit is reached scales directly with the coherence of the laser beam. So that limit is indeed the result of photon shot noise - and the photons are the places where an otherwise completely coherent wave has a local loss of coherence - a "phase slip".

In our coherent optical world, photons are the "noise" in an otherwise noiseless process. 
It is often argued that photon shot noise is observed in all laser beams, no matter how coherent they are. That statement is true, but it is not a statement about the laser beam, but rather about the detection process. We routinely observe photonic phenomena through the signals from incoherent detectors - photodiodes, phototransistors, etc. In an ordinary semiconductor photodiode, for example, the phases of the electron wave functions that are excited by the coherent beam have no relation to the phase of the beam. So each excitation event happens when a particular phase-matching condition occurs, independent of the other events. The result is a nearly-random stream of "photon" excitation events, each manifest as a pulse of electrical charge at the output of the detector. This stream of electrical signals thus exhibits "shot noise", which is mistakenly attributed to the statistics of "photon quantization" in the incoming laser beam.

Compare that phenomenon with the operation of a semiconductor laser, in which exquisite care is exercised to align the phase of the inverted electron population with that of the optical field. In the limit, the propagation vector of the electron wave function is identical to the propagation vector of the optical vector potential, the electrons form a collective quantum state, and no noise of any kind is present in either the beam or the laser current.

However, if the laser beam is "observed" by an incoherent detector, random "photon" absorption events in the detector disrupt the coherence in the beam, which is then reflected into the wave function of the inverted population in the laser, and thence into the laser current. So by "observing" the wave function, we have "collapsed" the wave function into a random set of "photon" events. What a horrible thing to do to an otherwise beautiful coherent system!

We are at the stage of detector evolution corresponding to the era of "crystal sets" in the evolution of radio receivers. We have already developed several forms of distributed coherent optical amplification. In the near future we will surely evolve more and more coherent forms of detection, which will then allow the kind of coherent systems at optical wavelengths that we routinely enjoy at radio wavelengths. The development of such coherent detectors has been seriously retarded by the Copenhagen view of laser beams made up of "photons"-little bullets

carrying $h \nu$ of energy. Einstein got past that hangup, and we can as well. At that point the "Copenhagen photon" will take its proper place with all the other "noise" bequeathed us by the Copenhagen clan!

\section{REFERENCES}

1. Carver Mead, Collective Electrodynamics: Quantum Foundations of Electromagnetism, MIT Press, Cambridge, Mass., 2000.

2. G. N. Lewis, Proceedings National Academy Science 12, p. 24, 1926.

3. H. Nyquist, Phys. Rev. 32, p 110, 1928.

4. H. Callen and T. Welton, Phys. Rev. 83, p. 34, 1951.

5. John G. Cramer, Reviews of Modern Physics 58, p. 647, 1986.

6. http://faculty.washington.edu/jcramer/theory.html 